\section{Avaliação do consumo alimentar de crianças brasileiras assistidas em creches: uma revisão sistemática}

\section{Evaluation of food consumption among Brazilian children attending daycare centers: a systematic review}

Dixis Figueroa Pedraza 1

Daiane de Queiroz 2

Jacqueline Santos da Fonsêca Almeida Gama 3

\begin{abstract}
Objectives: to provide evidence of the pattern of food consumption among Brazilian children attending daycare centers.

Methods: the PubMed, LILACS and SciELO databases were searched for observational studies published between 1990 and 2013 using the terms "food consumption" AND "child day care centers".

Results: fifty-eight articles were found, of which 21 were considered relevant to the present study. These included 18 cross-sectional and three longitudinal studies. The studies tended i) to be concentrated in the Southeast region of the country; ii) to use direct weighing of food and reference values from Dietary Reference Intakes to assess food consumption; iii) to present a preliminary overview, focused on the Southeast, of a pattern of consumption low on fruit and vegetables and with inadequate iron intake; iv) to provide results restricted to mean intake, owing to the lack of use of methods appropriate for establishing patterns of food intake.

Conclusions: despite the paucity and geographical dispersion of the studies, methodological similarities made it possible to suggest that there is a predominant pattern of inadequate consumption of fruit and vegetables and food rich in iron, along with excessive consumption of protein and high sodium contents.
\end{abstract}

Key words Food consumption, Child nutrition, Child day care centers
1Programa de Pós-Graduação em Saúde Pública. Departamento de Enfermagem. Universidade Estadual da Paraíba. Av. das Baraúnas, 351. Campus Universitário. Bodocongó. Campina Grande, PB, Brasil. CEP: 58.109-753. E-mail: dixisfigueroa@gmail.com

2 Faculdade de Ciências Médicas de Campina Grande. Campina Grande, PB, Brasil.

3 Departamento de Enfermagem. Universidade Estadual da Paraíba. Campina Grande, PB, Brasil

\section{Resumo}

Objetivos: levantar evidências sobre o perfil do consumo alimentar de crianças brasileiras assistidas em creches.

Métodos: foi realizada uma busca por estudos observacionais nas bases de dados PubMed, LILACS e SciELO. Foram selecionados artigos publicados entre 1990 e 2013, utilizando os termos "food consumption" AND "child day care centers".

Resultados: foram identificados 58 artigos, dos quais 21 artigos foram considerados relevantes para o presente trabalho: 18 estudos transversais e três longitudinais. A sistematização dos estudos destaca: i) a concentração geográfica no Sudeste do país; ii) a apropriação da pesagem direta de alimentos e dos valores de referência das Dietary Reference Intakes na avaliação do consumo de alimentos; iii) um panorama preliminar, com ênfase no Sudeste, do consumo deficitário de legumes, frutas e vegetais, $e$ da inadequação da ingestão dietética de ferro; iv) a restrição dos resultados à ingestão média devido à insuficiência do uso de métodos apropriados ao estabelecimento de padrões alimentares.

Conclusões: apesar da escassez e dispersão espaço-temporal dos estudos, as semelhanças metodológicas possibilitam sugerir um panorama em que predomina a ingestão dietética deficitária do grupo de frutas, legumes e verduras, e de alimentos ricos em ferro; bem como a ingestão excessiva de alimentos protéicos e de alto teor de sódio.

Palavras-chave Consumo de alimentos, Nutrição da criança, Creches 


\section{Introdução}

A medida da dieta tem uma ampla aplicação em vários campos de conhecimento na área de saúde. ${ }^{1}$ Os inquéritos alimentares possibilitam analisar a associação da dieta com o estado nutricional e com a situação de saúde.2,3 O conhecimento gerado por meio dessa análise evidencia a situação da adequação da ingestão alimentar e possibilita a definição de políticas públicas direcionadas a garantia da promoção da saúde.1-3

Diversos fatores interferem e tornam difícil o ato de registrar a ingestão de alimentos de um indivíduo, sem exercer influência sobre esse, dentre os quais a complexidade da dieta, os hábitos alimentares, a memória do entrevistado, a cultura e a situação socioeconômica. ${ }^{2}$ Nas crianças, apresenta-se como dificuldade adicional a incapacidade de reportar o consumo de alimentos, tornando-se imprescindível a participação dos seus responsáveis.2,4

Para muitas famílias as creches representam a oportunidade das crianças terem suas necessidades alimentares garantidas em um ambiente seguro, uma vez que a alimentação representa uma das grandes responsabilidades dessas instituições. 5 As crianças que frequentam creches devem receber, gratuitamente, no mínimo, $30 \%$ das suas necessidades diárias de alimentos, como direito instituído por meio do Programa Nacional de Alimentação Escolar, 6 favorecendo à elas um desenvolvimento com saúde intelectual e física. 5 Dessa forma, a avaliação do consumo alimentar desse grupo de indivíduos representa uma atividade de grande relevância.2,5

As creches são hoje uma realidade na vida de uma grande parcela das crianças brasileiras. A grande procura por estes serviços pode estar relacionada à crescente participação das mulheres no mercado de trabalho, 5 o que pode comprometer o tempo dispensado ao cuidado dos filhos.7,8 Conforma-se, assim, um quadro de responsabilização compartilhada entre os meios institucional e o familiar com relação a alimentação infantil, 5 numa relação complexa com perspectivas diferentes sobre o desenvolvimento e as suas necessidades, capazes de comprometer o cuidado com a criança. ${ }^{9}$

Observa-se, deste modo, que as crianças assistidas em creches encontram-se inseridas numa conjuntura de múltiplas influências familiares, sociais e ambientais, relacionadas, determinantes do padrão alimentar. ${ }^{8}$ Face ao exposto, o objetivo deste trabalho foi levantar evidências sobre o perfil do consumo alimentar de crianças brasileiras assistidas em creches.

\section{Métodos}

A revisão da literatura foi realizada a partir de um levantamento bibliográfico nas bases de dados PubMed (National Library of Medicine, Bethesda, MD), LILACS (Literatura Latino-americana e do Caribe em Ciências da Saúde) e SciELO (Scientific Electronic Library Online). Na busca utilizaram-se os termos "food consumption" AND "child day care centers" e seus equivalentes em português e espanhol. No caso das buscas no PubMed, considerando a grande quantidade de registros fora do foco da revisão, o descritor Brazil também foi usado. Os estudos publicados a partir de 1990 foram investigados, aceitando-se aqueles escritos em português, inglês e espanhol. A busca foi realizada em 09/02/2014 por dois revisores de forma independente.

Para o cômputo do total de estudos identificados, foi verificada a duplicação dos mesmos entre as bases de dados, sendo cada artigo contabilizado somente uma vez. A decisão sobre a inclusão dos artigos incluiu duas etapas: i) triagem por meio da leitura dos títulos e resumos, ii) leitura na íntegra. $\mathrm{Na}$ fase de triagem, foram eliminados estudos de intervenção, registros tipo livro ou tese, estudos realizados fora do Brasil e artigos de revisão. Na fase de leitura na íntegra, optou-se por incluir estudos que analisaram indicadores de ingestão alimentar de crianças brasileiras assistidas em creches (todos os tipos de creches). Os estudos sem análise de indicadores da ingestão alimentar foram excluídos.

Os estudos incluídos foram caracterizados segundo autor e ano de publicação, objetivos, participantes/ população, faixa etária, tamanho da amostra e perdas, métodos de avaliação dietética utilizados, recomendações nutricionais para o diagnóstico, e principais resultados. Os estudos de delineamento transversal foram avaliados em relação à qualidade metodológica do tamanho da amostra. Entretanto, decidiu-se por não excluir aqueles identificados com problemas, considerando o interesse do presente trabalho nos métodos de avaliação do consumo alimentar utilizados pelos autores.

\section{Resultados}

Foram identificados 76 registros nas bases de dados pesquisadas, dos quais 18 estavam duplicados, totalizando 58 registros que foram submetidos à triagem. Após análise dos títulos e resumos, foram excluídos 25 registros que não preenchiam os critérios de seleção. Posteriormente, com a leitura na íntegra dos 33 artigos elegíveis, 12 foram excluídos por não 
utilizarem indicadores de ingestão alimentar, sendo incluídos, portanto, 21 artigos para sistematização. O fluxo relacionado à identificação e seleção dos estudos encontra-se na Figura 1.

A Tabela 1 mostra a distribuição dos estudos quanto aos parâmetros de caracterização adotados. Dos 21 artigos incluídos, 10-30 18 apresentam delineamento transversal11-18,21-30 e os outros três são estudos longitudinais. 10,19,20 A distribuição geográfica mostra concentração dos estudos na Região Sudeste ( $\mathrm{n}=8) .10,11,19-21,24,25,28$ Os demais, foram realizados nas Regiões Nordeste $(\mathrm{n}=5) ; 14,15,22,26,27$ Norte $(\mathrm{n}=3) ; 12,29,30 \mathrm{Sul}(\mathrm{n}=3)^{13,16,18}$ e Centro-Oeste $(\mathrm{n}=2) \cdot 17,23$ No Sudeste, Rio de Janeiro, 10,19,20 São Paulo11,24,25 e Minas Gerais 21,28 foram os Estados mais representados. No Nordeste, Pernambuco15,22 e Piauí26,27 concentraram a maioria dos estudos. Os estudos na Região Centro-Oeste e no Norte do país foram desenvolvidos no Distrito Federal e no Amazonas, respectivamente, enquanto no Sul apenas Rio Grande do Sul13,16 foi considerado em mais de um trabalho.

A faixa etária das crianças apresentou comportamento heterogêneo. Considerando a idade em anos completos, observa-se que quase todos os estudos $(n=20)$ envolveu crianças de até seis anos de idade1025,27-30 e que sete deles incluíram crianças menores de dois anos.11,14,17,22-24,29 O tipo de instituição foi diverso, tendo sido realizados, por exemplo, sete estudos em creches públicas 10,14,15,22,26-28 e seis em instituições filantrópicas ou administradas por entidades não governamentais.17-20,25,29 Em três estudos 21,23,24 não foi informado o tipo de instituição.

O tamanho da amostra variou, incluindo menos de 100 crianças $(n=6) ; 10,18-20,21,25$ de 100 a 300 crianças $(n=7)^{11,14,22-24,28,30}$ e acima de 300 crianças $(\mathrm{n}=7) .12,13,15-17,26,27$ Perdas amostrais expressivas foram reportadas nos estudos de Araújo et al. 26 e de Nagahama et al.30 Os estudos de menor tamanho amostral foram os de desenho longitudinal.10,19,20 O realizado por Yuyama et al. ${ }^{29}$ não referiu o tamanho da amostra. A avaliação da qualidade metodológica do tamanho da amostra dos estudos de delineamento transversal indicou problemas em cinco deles. $18,21,24,25,30$

Somente dois estudos 14,29 não utilizaram a pesagem direta dos alimentos como método de avaliação dietética. Entre os pesquisadores que relataram a quantidade de dias para a pesagem direta dos alimentos, vê-se que o procedimento foi realizado três vezes em seis estudos, $10,11,13,17,21,23$ duas vezes em dois estudos 19,20 e cinco vezes em um estudo. 27 O recordatório de 24 horas foi utilizado em oito estudos, $14,15,17,21-23,25,30$ o registro alimentar em seis, $10,12,13,16,19,20$ o questionário de frequência alimentar em três $21,23,26$ e a história dietética em dois. 19,20

Para a avaliação da adequação da ingestão dietética, observa-se que os pesquisadores usaram recomendações internacionais e nacionais, incluindo as recomendações da Organização das Nações Unidas para Alimentação e Agricultura/Organização Mundial da Saúde (FAO/OMS) de 1974,31 as recomendações para energia e proteínas da OMS de 1985,32 as Necessidades Médias Estimadas de 198933 e as recomendações da Sociedade Brasileira de Alimentação e Nutrição de $1990 .{ }^{34}$ As Dietary Reference Intakes (DRI) ${ }^{35}$ foram utilizadas em vários estudos, incluindo os valores de Estimated Average Requirement (EAR) e de Adequate Intake (AI), referenciados pelo Food and Nutrition Board do Institute of Medicine.36-39 Os parâmetros das DRI publicadas no ano de 199740 também foram usados. Considerando os estudos que divulgaram resultados sobre o consumo por grupos de alimentos, o leite e seus derivados $21,23,25,27$ e os alimentos açucarados,21,23,29 foram citados como os de maior consumo em vários destes. Vegetais folhosos, legumes e frutas foram reportados como alimentos de consumo insatisfatório em cinco dos manuscritos.20,21,24,25,27 A mesma situação foi reportada em três trabalhos $23,25,27$ para peixes, fígado e miúdos. Nenhum dos estudos citados representou a Região Sul do país, bem como, somente para o grupo do leite (consumo satisfatório) e das verduras/frutas/ legumes (consumo insatisfatório), verifica-se a citação de no mínimo dois estudos de uma mesma Região (Sudeste). Fernandes et al.22 e Araújo et $a l .,{ }^{26}$ no Nordeste, investigando a ingestão dietética de vitamina A, relataram menor consumo de vegetais fontes desta vitamina em detrimento dos alimentos de origem animal.

Nos estudos que divulgaram resultados sobre a ingestão dietética de nutrientes e energia, os nutrientes mais frequentemente citados como de "consumo acima das recomendações" foram a proteína $11,12,17,18,21,24$ e o sódio.12,18,29 O ferro $11,21,24,29,30$ e a energia, $11,21,24,25,30$ por sua vez, tiveram a maior quantidade de reportes como de "consumo abaixo das recomendações". Nesses estudos, considerando aqueles que analisaram a adequação de nutrientes, os valores oscilaram de $106,7 \% 18$ a $189,6 \% 24$ para a proteína; $167,6 \% 18$ a $512,0 \% 29$ para o sódio; $38,4 \% 24$ a $81,7 \% 11$ para o ferro e $36,5 \% 30$ a $58,9 \% 24$ para a energia (dados não disponíveis em tabela). Nenhum dos estudos elencados representou a Região Nordeste do país; foram 
encontrados, no mínimo, dois estudos com a citação da mesma Região: para proteína (acima das recomendações) no Sudeste; sódio (acima das recomendações) no Norte; ferro (abaixo das recomendações) no Sudeste e no Norte, e energia (abaixo das recomendações) no Sudeste.

Dos estudos que enfocaram a vitamina
A, 15,22,26,27 dois, 22,26 no Nordeste, revelaram um menor consumo dietético nas crianças de maior faixa etária e um 15 relatou consumo acima das recomendações para a maioria das crianças. Para o micronutriente zinco, relatou-se prevalência moderada de inadequação dietética. ${ }^{14}$

\section{Figura 1}

Fluxograma das fases de identificação, triagem e seleção de artigos sobre o consumo de alimentos de crianças brasileiras assistidas em creches.

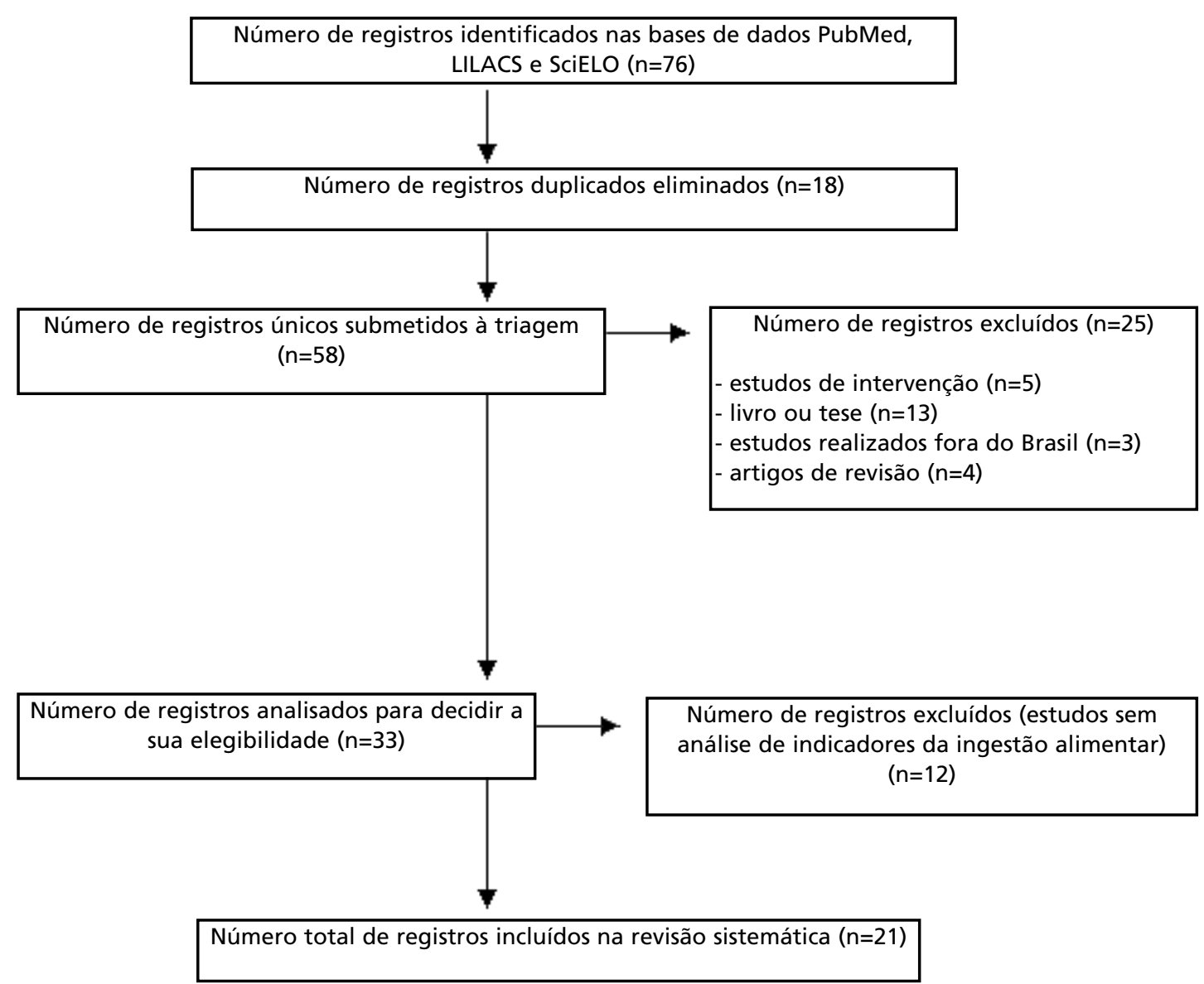




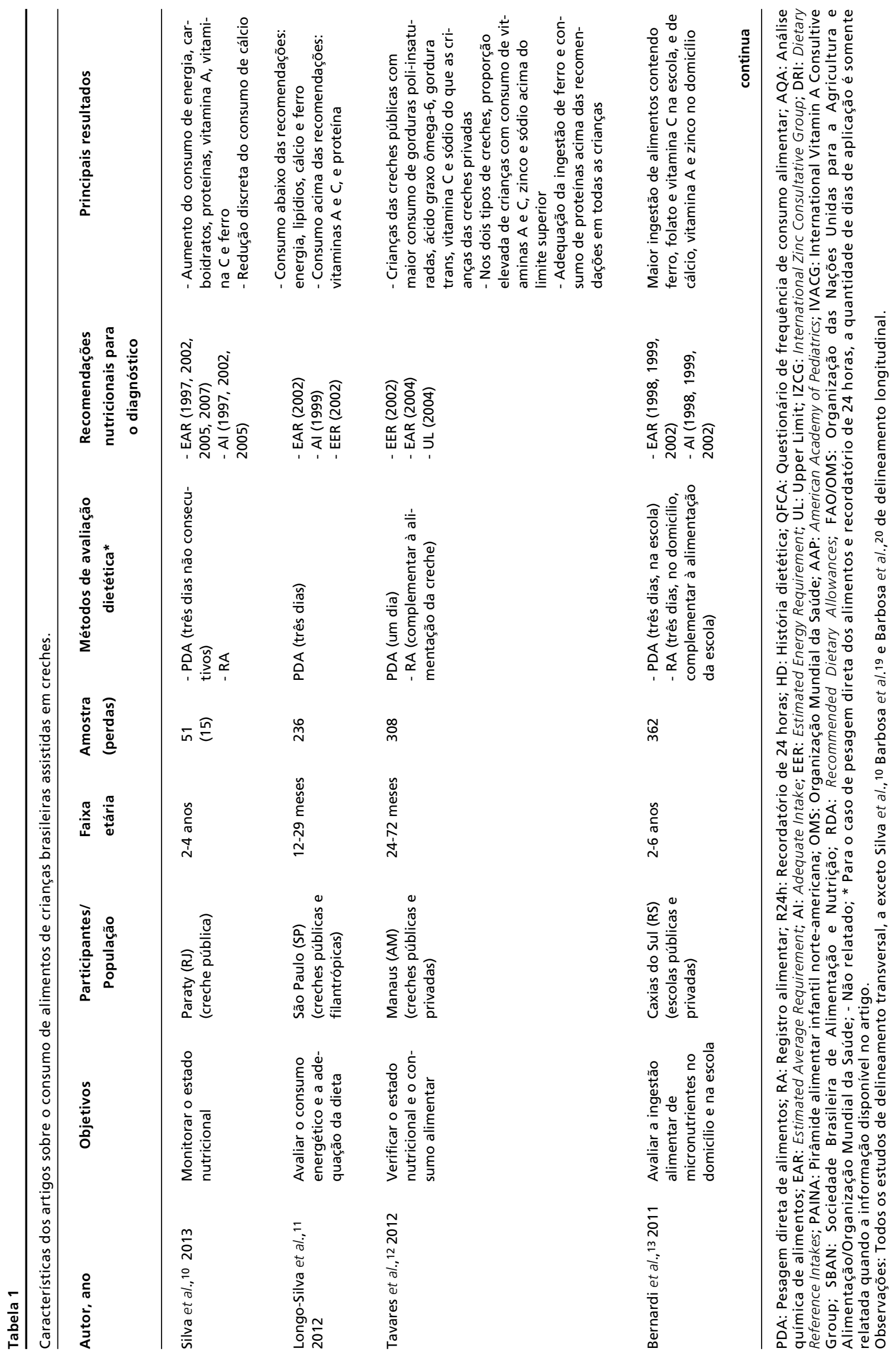




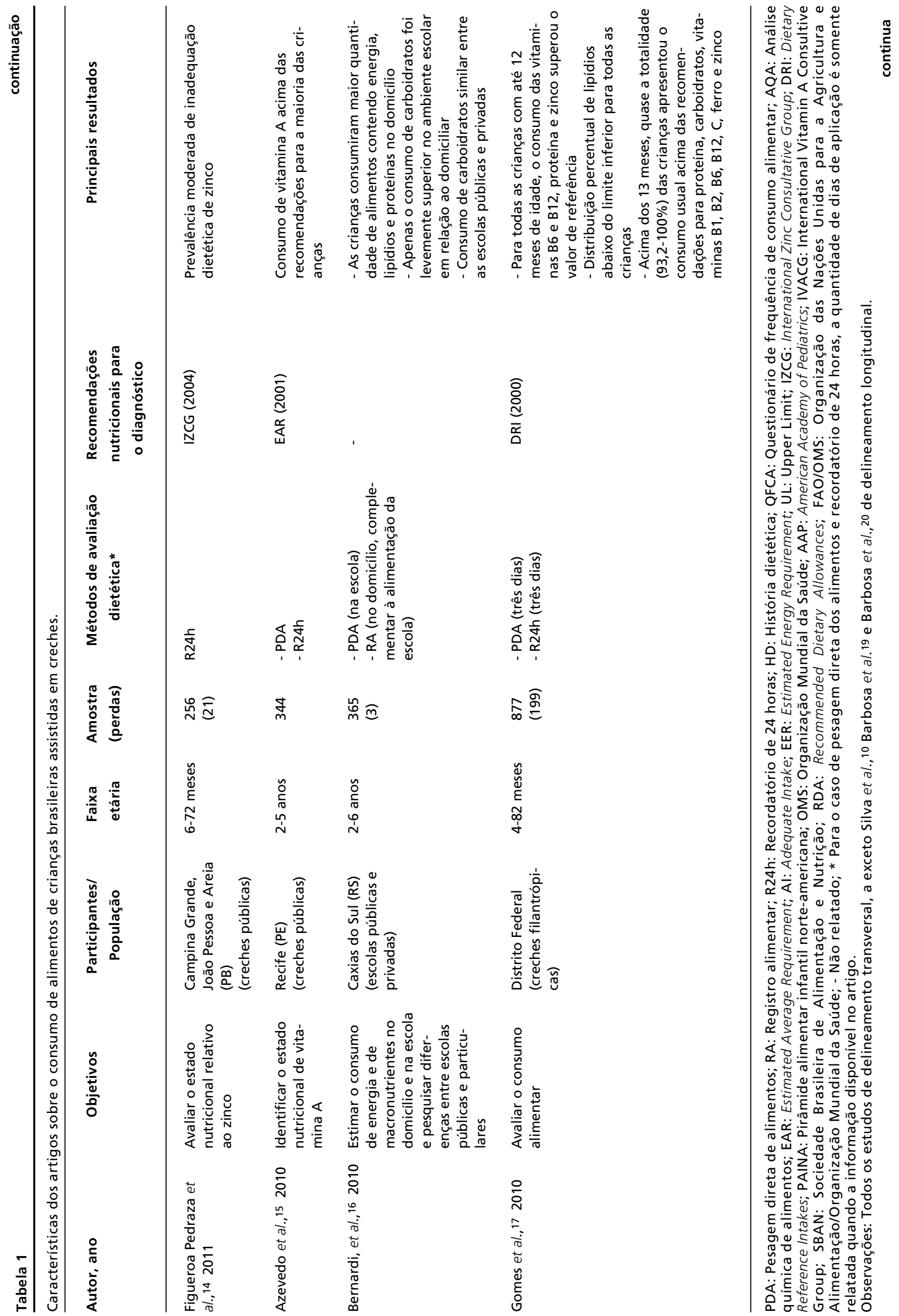




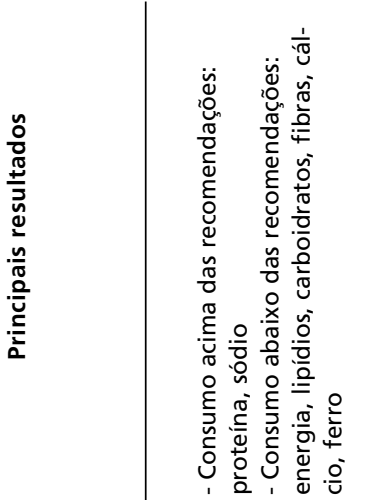

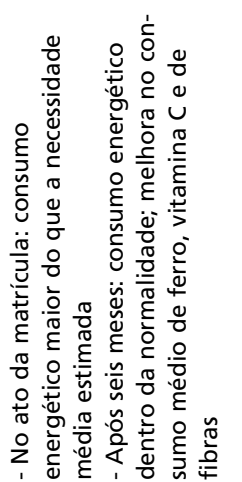

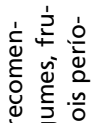

要这

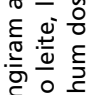

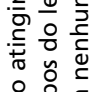

io

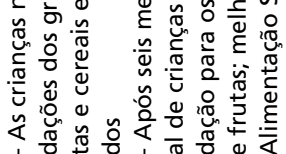

ब

융 용

节

产

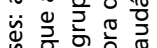

叫

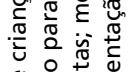

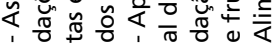

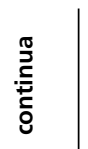

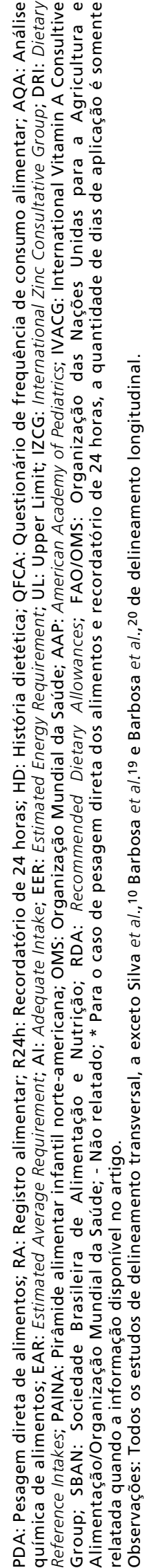




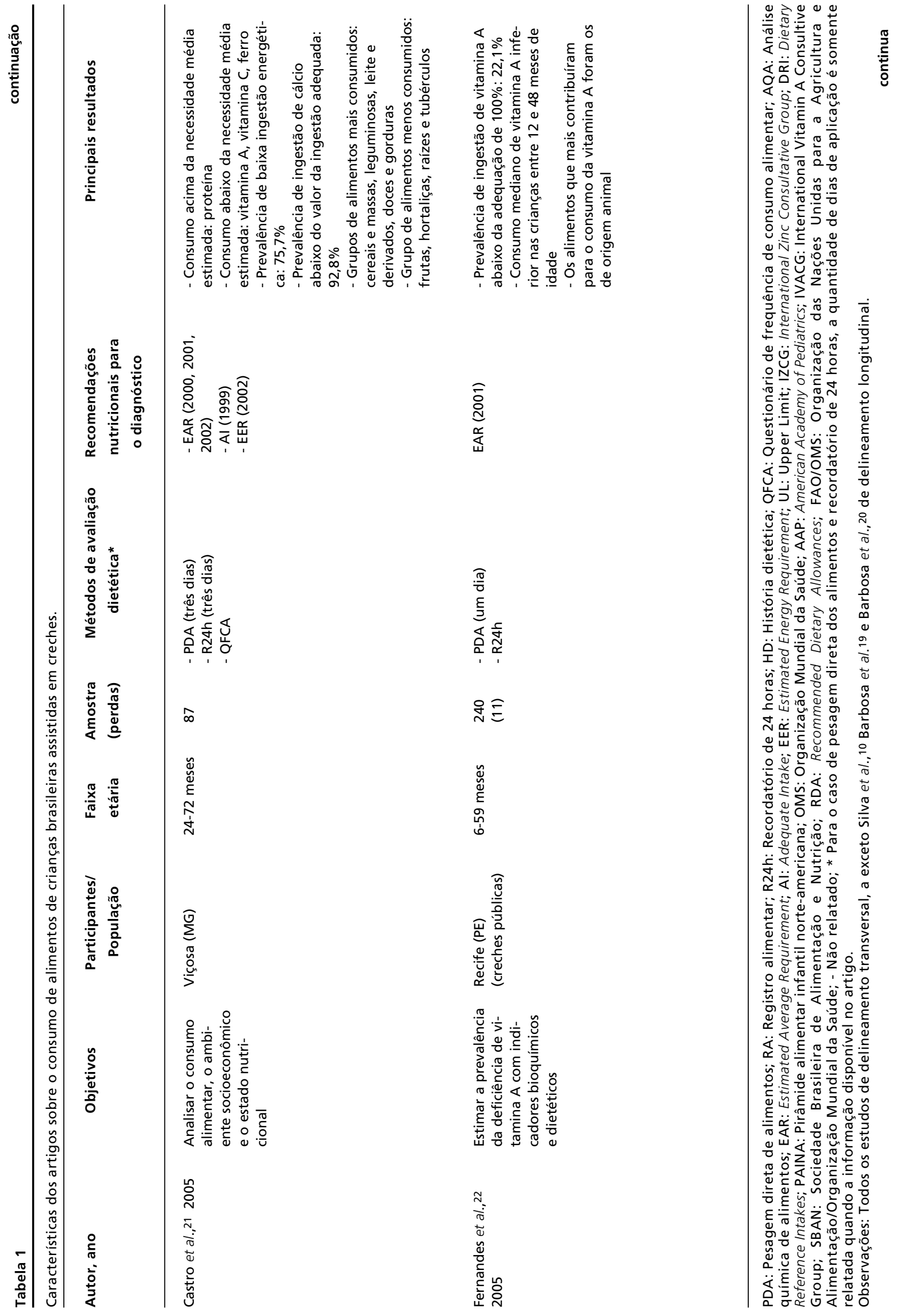




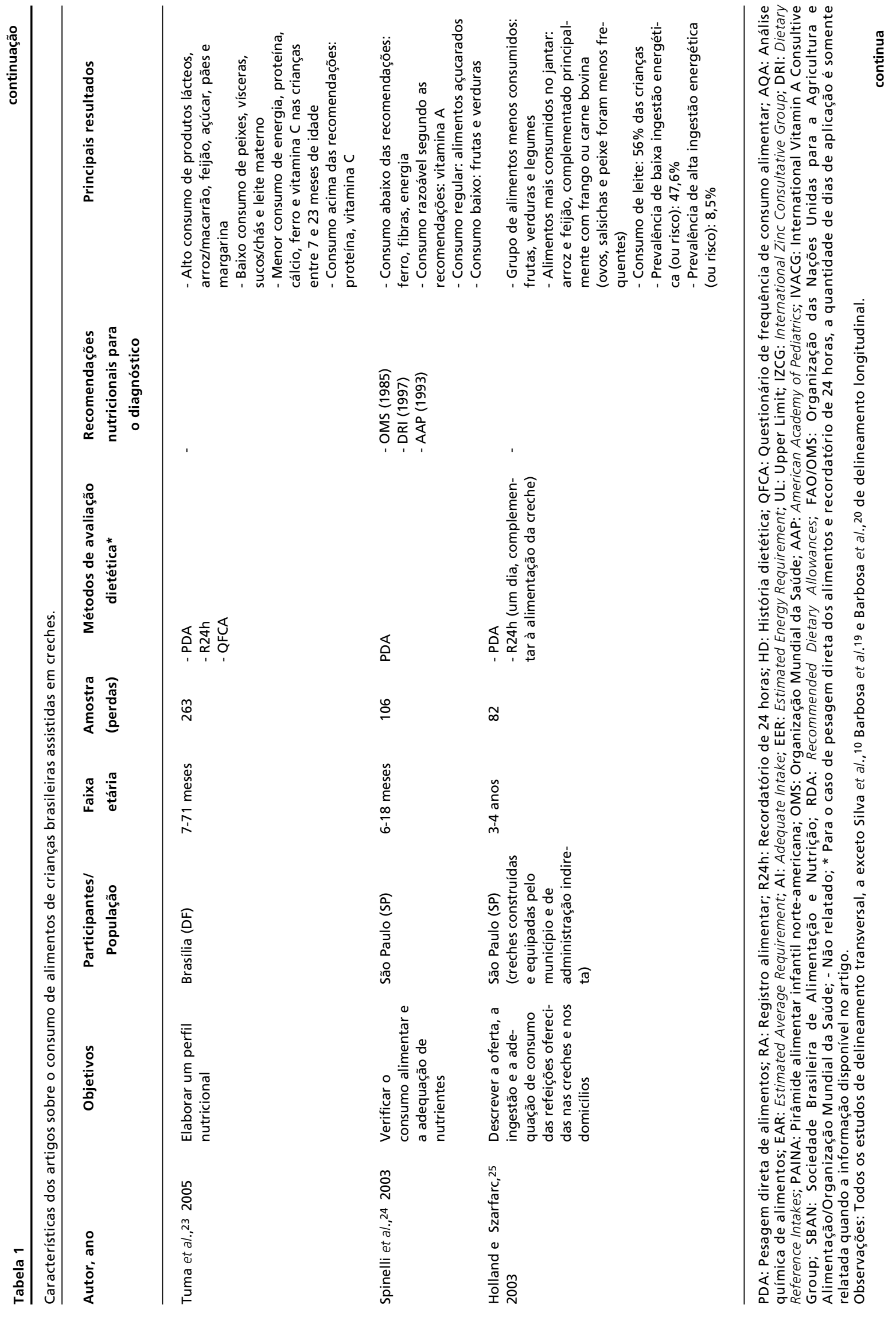

Rev. Bras. Saúde Matern. Infant., Recife, 15 (1): 17-31 jan. / mar., 2015 


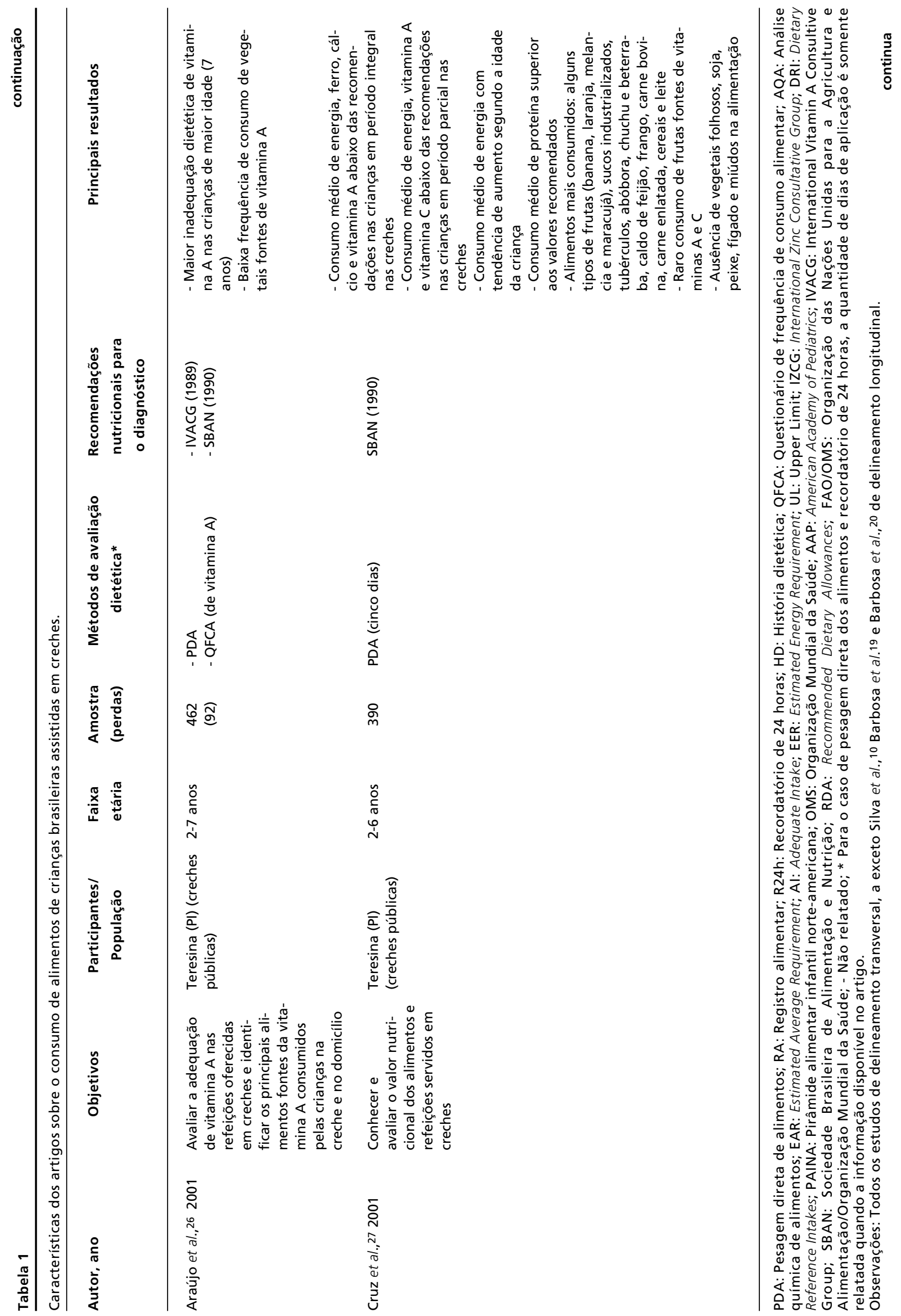




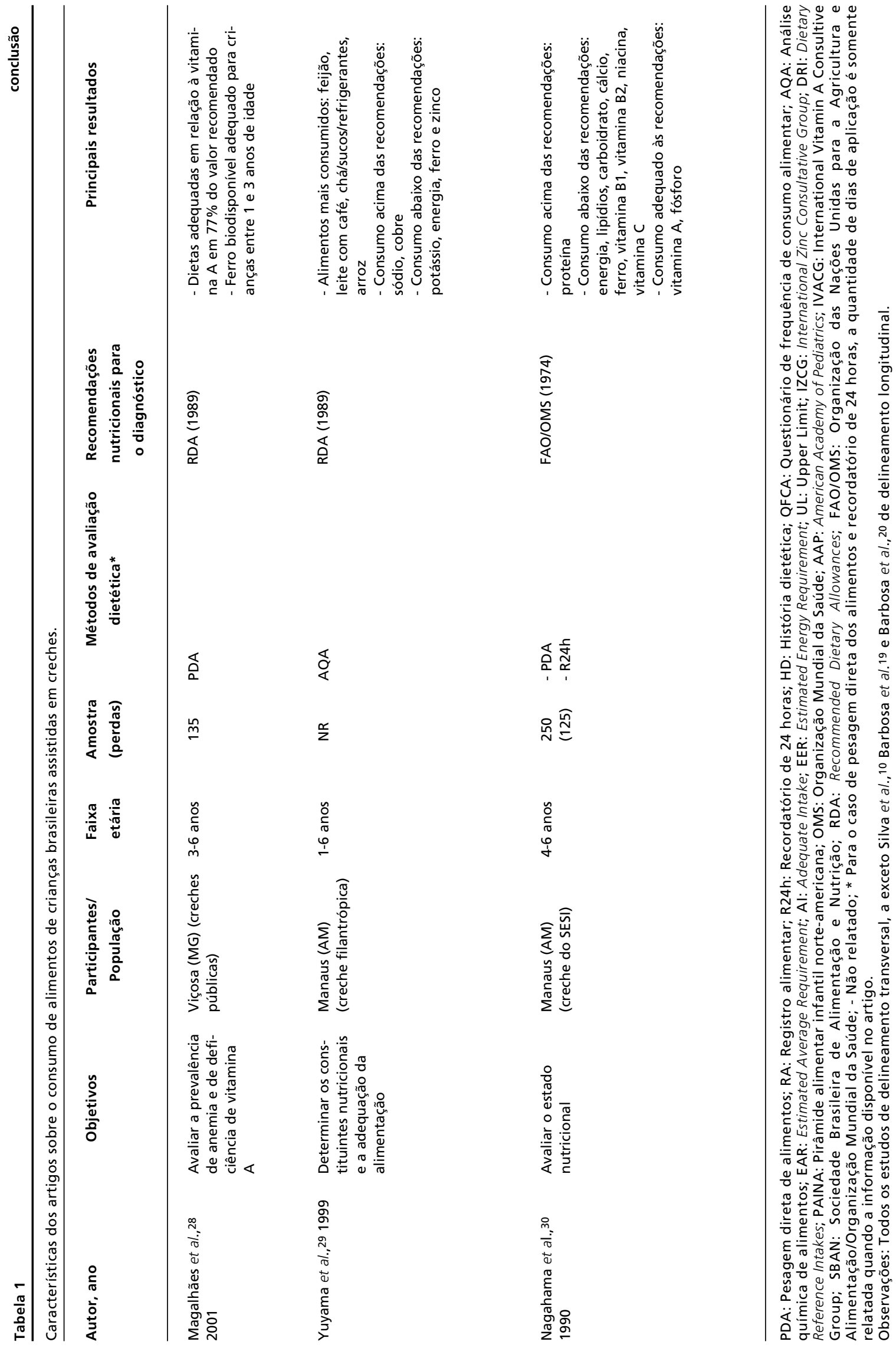




\section{Discussão}

Apesar de os resultados encontrados mostrarem que existe uma escassez de estudos sobre o consumo de alimentos em crianças brasileiras assistidas em creches, estando estes ainda concentrados geograficamente na Região Sudeste do país e com dispersão associada ao tempo (21 estudos no período de 1990 a 2013), as semelhanças metodológicas garantem a comparabilidade dos mesmos. Até o momento não existem artigos publicados sistematizando o conhecimento sobre o perfil relacionado ao consumo de alimentos desse grupo, servindo o presente artigo para apresentação, atualização e sistematização.

A escassez de estudos relacionados ao consumo de alimentos de crianças foi constatada em outras revisões.4,41 Este fato pode estar associado, além da complexidade própria de tal avaliação, 2 como já mencionado, às dificuldades para estabelecer associações entre a dieta e a ocorrência de doenças, que podem gerar vieses nas análises. Esses fatores exigem uma coleta de dados criteriosa e o uso de técnicas estatísticas apropriadas que possibilitem aproximar as informações relatadas pelos entrevistados com a real ingestão de nutrientes e de energia. ${ }^{3}$

A concentração da produção científica no Sudeste do Brasil vem sendo apontada por outros estudos de revisão sobre temas da área de saúde. ${ }^{42,43}$ É, portanto, necessário desenvolver uma maior quantidade de estudos que representem as desigualdades socioespaciais da população brasileira e as influências geradas pelos alimentos típicos e pela cultura alimentar das diferentes regiões do Brasil.

Segundo a literatura, dentre os principais vieses presentes nos estudos sobre consumo alimentar encontram-se aqueles inerentes ao tamanho amostral e ao processo de amostragem. ${ }^{3,4} \mathrm{O}$ aumento da amostra é necessário quando a variação interindividual, que varia para cada nutriente e depende da heterogeneidade da população, é grande. Ainda, considera-se que a obtenção de informações de um maior número de dias é uma alternativa para a obtenção de dados mais confiáveis nos casos de estudos com quantidade de indivíduos insuficientes. ${ }^{4}$ Assim, o tamanho de amostra inapropriado dos trabalhos que foram identificados com problemas nesse quesito $18,21,24,25,30$ compromete a generalização dos seus resultados, sobretudo naqueles em que o consumo de alimentos foi verificado exclusivamente em um dia. $18,24,25,30$

Em relação aos métodos de avaliação dietética usados pelos pesquisadores, destaca-se o uso preferencial da pesagem dos alimentos ingeridos. Este tem sido considerado o método mais exato para deter- minar a ingestão de alimentos, ${ }^{2}$ aspecto positivo não somente do ponto de vista metodológico assim como por tornar comparáveis os resultados dos estudos. Fica posto, então, que o uso da pesagem dos alimentos é factível no contexto das creches, devendo haver uma menor valorização das limitações que lhe são atribuídas, como ser demorado, oneroso e invasivo. ${ }^{2}$

O recordatório de 24 horas e o registro alimentar também foram métodos usados por vários pesquisadores, enquanto o questionário de frequência alimentar foi pouco utilizado. Considerando que, similarmente ao método de pesagem de alimentos, o recordatório de 24 horas e o registro alimentar avaliam a ingestão atual/ingestão média de alimentos e/ou nutrientes, e que a frequência alimentar descreve os padrões alimentares, 2,3,44 sugere-se uma lacuna no conhecimento sobre os hábitos alimentares das crianças assistidas em creches. Ainda não existe no Brasil nenhum questionário de frequência de consumo de alimentos validado para crianças menores de cinco anos, ${ }^{45}$ explicando esse resultado e indicando a necessidade de investimentos nesse sentido.

Para a avaliação da adequação da ingestão de nutrientes, observa-se a apropriação de valores de referência com a evolução dos métodos. Nos estudos mais antigos sobressai o uso das recomendações das décadas de 1970 e 1980, e das DRI naqueles mais recentes. Nesse sentido, é necessário ressaltar que a utilização dos valores de referências das DRI constitui a recomendação atual na avaliação de dietas e interpretação dos inquéritos alimentares, tendo na análise da EAR o parâmetro mais indicado para expressar a prevalência de inadequação da ingestão alimentar.

Saliente-se que o uso da Recommended Dietary Allowance (RDA) - ingestão dietética recomendada - não é recomendado na avaliação do consumo alimentar de grupos, pois pode resultar na superestimação da ingestão dietética.4,46 A aplicação das recomendações das DRI para a população brasileira deve ponderar os dados de ingestão dietética com o seu erro associado, pois as mesmas foram estabelecidas para as populações dos Estados Unidos e do Canadá. 46

Com relação aos resultados dos estudos revisados, o presente trabalho conseguiu sistematizar o consumo deficitário de legumes, frutas e vegetais. Esse consumo deficiente de alimentos ricos em nutrientes pode ser sugerido ao menos para as Regiões Sudeste (considerando os estudos com base no consumo por grupos de alimentos) e Nordeste (considerando os estudos com base na ingestão 
dietética de vitamina A). Situação similar apresentase com relação à ingestão acima das recomendações para a proteína, marcadamente no Sudeste, e o sódio, no Norte.

Estes achados estão em sintonia com o perfil nacional, que indica modificações no padrão alimentar com aumento no consumo de alimentos industrializados e redução do consumo de frutas, legumes e verduras. ${ }^{47}$ Este perfil pode contribuir para o desenvolvimento de obesidade, com repercussões na saúde das crianças e maior risco de doenças crônicas não transmissíveis na vida adulta, o que sinaliza a necessidade de promover a alimentação saudável, com o envolvimento da família, de órgãos governamentais e dos meios de comunicação. 48 Nesse contexto, o Programa Nacional de Alimentação Escolar é essencial para o cumprimento das normas do Programa.49,50

Os resultados deste estudo reforçam a carência de inquéritos alimentares, especificamente no que se refere a vitaminas e minerais, apontada anteriormente, 51 limitando a adoção de medidas preventivas considerando os indicadores dietéticos como primeira referência do risco de deficiências nutricionais.2 Os indícios de ingestão dietética deficitária em ferro nas Regiões Sudeste e Norte do país, encontrados, são comparáveis aos reportados em estudo de base populacional com crianças pernambucanas menores de cinco anos. 51

Conclui-se, a partir dos achados desta revisão: i)

\section{Referências}

1. Cardoso MA, Olinto MTA, Sichieri R. Avanços metodológicos em estudos populacionais em alimentação e nutrição. Cad Saúde Pública. 2010; 26 (11): 2006-7.

2. Cavalcante AAM, Priore SE, Franceschini SCC. Estudos de consumo alimentar: aspectos metodológicos gerais e o seu emprego na avaliação de crianças e adolescentes. Rev Bras Saúde Matern Infant. 2004; 4: 229-40.

3. Costa AGV, Priore SE, Sabarense CM, Franceschini SCC Questionário de freqüência de consumo alimentar e recordatório de 24 horas: aspectos metodológicos para avaliação da ingestão de lipídeos. Rev Nutr. 2006; 19 (5): 631-41.

4. Falcão-Gomes RC, Coelho AAS, Schmitz BAS Caracterização dos estudos de avaliação do consumo alimentar de pré-escolares. Rev Nutr. 2006; 19 (6): 713-27.

5. Goulart RMM, Banduk MLS, Taddei JAAC. Uma revisão das ações de nutrição e do papel do nutricionista em creches. Rev Nutr. 2010; 23 (4): 655-65

6. Peixinho A, Balaban D, Rimkus L, Schwartzman F, Galante AP. Alimentação Escolar no Brasil e nos Estados Unidos. Mundo Saúde. 2011; 35 (2): 128-36.

7. Leal VS, Lira PIC, Oliveira JS, Menezes RCE, Sequeira LAS, Arruda Neto MA, Andrade SLLS, Batista Filho M. a escassez de pesquisas e dispersão espaço-temporal com concentração na Região Sudeste; ii) o uso preferencial da pesagem direta de alimentos e a incorporação dos valores de referência das DRI como métodos de avaliação; iii) o consumo deficitário de legumes, frutas e vegetais, ao menos nas Regiões Sudeste e Nordeste; iv) a escassez de resultados sobre a adequação dietética de vitaminas e minerais com indícios de dietas deficitárias em ferro, ao menos nas Regiões Sudeste e Norte; v) sinais de consumo acima das recomendações de proteína na Região Sudeste e de sódio na Região Norte; vi) a restrição dos resultados à ingestão média devido à insuficiência do uso de métodos apropriados ao estabelecimento de padrões alimentares.

São necessários, portanto, investimentos em pesquisa, visando o desenho e validação de instrumentos e questionários de frequência de consumo de alimentos, por exemplo, que tornem possível traçar os hábitos alimentares e a adequação dietética de micronutrientes de crianças. Ainda, considerando a concentração da produção científica na Região Sudeste do país, há necessidade de pesquisas direcionadas a melhorar o conhecimento sobre o consumo de alimentos das crianças brasileiras assistidas em creches. Tais recomendações são importantes, inclusive, para análises referentes à execução do Programa Nacional de Alimentação Escolar.

Excesso de peso em crianças e adolescentes no Estado de Pernambuco, Brasil: prevalência e determinantes. Cad Saúde Pública. 2012; 28 (6): 1175-82.

8. Rossi A, Moreira EAM, Rauen MS. Determinantes do comportamento alimentar: uma revisão com enfoque na família. Rev Nutr. 2008; 21 (6): 739-48.

9. Maranhão DG, Sarti CA. Creche e família: uma parceria necessária. Cad Pesq. 2008; 38 (133): 171-94.

10. Silva PA, Araújo PNG, Firmino NNJ, Serrão LH, Abreu SE. Estado nutricional de pré-escolares de creche pública: um estudo longitudinal. Cad Saúde Colet. 2013; 21 (2): 140-7.

11. Longo-Silva G, Toloni MHA, Goulart RMM, Taddei JAA. Avaliação do consumo alimentar em creches públicas em São Paulo, Brasil. Rev Paul Pediatr. 2012; 30 (1): 35-41.

12. Tavares BM, Veiga GV, Yuyama LKO, Bueno MB, Fisberg RM, Fisberg M. Estado nutricional e consumo de energia e nutrientes de pré-escolares que frequentam creches no município de Manaus, Amazonas: existem diferenças entre creches públicas e privadas? Rev Paul Pediatr. 2012; 30 (1): 42-50.

13. Bernardi JR, Cezaro C, Fisberg RM, Fisberg M, Rodrigues GP, Vitolo MR. Consumo alimentar de micronutrientes 
entre pré-escolares no domicílio e em escolas de educação infantil do município de Caxias do Sul (RS). Rev Nutr. 2011; 24 (2): 253-61

14. Figueroa Pedraza D, Rocha ACD, Queiroz EO, Sousa CPC. Estado nutricional relativo ao zinco de crianças que frequentam creches do estado da Paraíba. Rev Nutr. 2011; 24 (4): 539-52

15. Azevedo MMS, Cabral PC, Diniz AS, Fisberg M, Fisberg RM, Arruda IKG. Deficiência de vitamina A em pré-escolares da cidade do Recife, Nordeste do Brasil. Arch Latinoam Nutr. 2010; 60 (1): 36-41.

16. Bernardi JR, Cezaro C, Fisberg RM, Fisberg M, Vitolo MR. Estimation of energy and macronutrient intake at home and in the Kindergarten programs in preschool children. J Pediatr. 2010; 86 (1): 50-64.

17. Gomes RCF, Costa THM, Schmitz BAS. Avaliação do consumo alimentar de pré-escolares do Distrito Federal, Brasil. Arch Latinoam Nutr. 2010; 60 (2): 168-74.

18. Alves G, Colauto ÉV, Fernandes JK, Zabine L, Nienow RC. Avaliação antropométrica e consumo alimentar de pré-escolares em creches de Umuarama, Paraná. Arq Ciênc Saúde Unipar. 2008; 12 (2): 119-26.

19. Barbosa RMS, Soares EA, Lanzillotti HS. Avaliação da ingestão de nutrientes de crianças de uma creche filantrópica: aplicação do Consumo Dietético de Referência. Rev Bras Saúde Matern Infant. 2007; 7 (2): 159-66.

20. Barbosa RMS, Carvalho CGN, Franco VC, Salles-Costa R, Soares EA. Avaliação do consumo alimentar de crianças pertencentes a uma creche filantrópica na Ilha de Paquetá, Rio de Janeiro, Brasil. Rev Bras Saúde Matern Infant. 2006; 6 (1): $127-34$.

21. Castro TG, Novaes JF, Silva MR, Costa NMB, Franceschini SCC, Tinôco ALA, Leal PFG. Caracterização do consumo alimentar, ambiente socioeconômico e estado nutricional de pré-escolares de creches municipais. Rev Nutr. 2005; 18 (3): $321-30$.

22. Fernandes TFS, Diniz AS, Cabral PC, Oliveira RS, Lóla MMS, Silva SMM, Kolsteren P. Hipovitaminose A em préescolares de creches públicas do Recife: indicadores bioquímico e dietético. Rev Nutr. 2005; 18 (4): 471-80.

23. Tuma RCFBT, Costa THM, Schmitz BAS. Avaliação antropométrica e dietética de pré-escolares em três creches de Brasília, Distrito Federal. Rev Bras Saúde Matern Infant. 2005; 5 (4): 419-28.

24. Spinelli MGN, Goulart RMM, Santos ALP, Gumiero LDC, Farhud CC, Freita EB, Dantas. Consumo alimentar de crianças de 6 a 18 meses em creches. Rev Nutr. 2003; 16 (4): 409-14

25. Holland CV, Szarfarc SC. Consumo energético do préescolar de creches. Nutrire Rev Soc Bras Aliment Nutr. 2003; $25: 61-70$

26. Araújo KC, Carvalho CMRG, Paz SMRS. Avaliação do consumo alimentar de vitamina A de crianças assistidas em creches comunitárias, Teresina (PI), Brasil. Nutrire Rev Soc Bras Aliment Nutr. 2001; 22: 7-19.

27. Cruz GF, Santos RS, Carvalho CMRG, Moita GC. Avaliação dietética em creches municipais de Teresina, Piauí, Brasil. Rev Nutr. 2001; 14 (1): 21-32.
28. Magalhães P, Ramalho AR, Colli C. Deficiência de ferro e de vitamina A: avaliação nutricional de pré-escolares de Viçosa (MG/Brasil). Nutrire Rev Soc Bras Aliment Nutr. 2001; 21: 41-56.

29. Yuyama LKO, Vasquez ALV, Aguiar JPL, Macedo SHM, Yonekura L, Nagahama D, Fonseca CW. Composição química e adequação da alimentação oferecida aos préescolares de uma instituição beneficente de Manaus, Amazonas, Brasil. Acta Amaz. 1999; 29 (4): 549-54.

30. Nagahama D, Marinho HA, Rocha Y, Ferraroni MJR, Silva NB, Castro JS, Onety JA. Avaliação nutricional e alimentar de pré-escolares de uma creche de Manaus e a influencia da entidade no estado nutricional de sua população. Acta Amaz. 1990; 20: 119-29.

31. FAO/OMS (Organización de las Naciones Unidas para la Agricultura y Alimentación, Organización Mundial de la Salud). Manual sobre las necesidades del hombre. Roma; 1974

32. OMS (Organización Mundial de la Salud). Necesidades de energía y de proteínas. Ginebra: FAO/OMS/UNU; 1985. 220 p. (Série de Informes Técnicos 724).

33. National Research Council, Food and Nutrition Board. Recommended Dietary Allowances. 10 ed. Washington: National Academy Press; 1989. 284p.

34. Vannucchi H, Meneses EW, Camapana AO, Lajolo FM. Aplicações das recomendações nutricionais adaptadas à população brasileira. Cad Nutr. 1990; 2: 102-5.

35. Institute of Medicine. Food and Nutrition Board. Dietary References Intakes: Applications in Dietary Assessment. Washington, DC: National Academy Press; 2000.

36. Institute of Medicine. Food and Nutrition Board. Dietary References Intakes for calcium, phosphorus, magnesium, vitamin D, and fluoride. Washington, DC: National Academies Press; 1999.

37. Institute of Medicine. Food and Nutrition Board. Dietary References Intakes for vitamin C, vitamin E, selenium, and carotenoids. Washington, DC: National Academies Press; 2000 .

38. Institute of Medicine. Food and Nutrition Board. Dietary References Intakes for vitamin A, vitamin K, arsenic, boron, chomium, copper, iodine, iron, manganese, molybdenum, nickel, silicon, vanadium, and zinc. Washington, DC: National Academies Press; 2001.

39. Institute of Medicine. Food and Nutrition Board. Dietary References Intakes for energy, carboydrate, fiber, fat, fatty acids, cholesterol, protein and amino acids (macronutrients). Washington, DC: National Academies Press; 2002.

40. Institute of Medicine. Food and Nutrition Board. Dietary References Intakes: recommended intakes for individuals, vitamins, foods and nutrition board. Washington, DC: National Academy of Sciences; 1997.

41. Pereira AS, Lanzillotti HS, Soares EA. Frequência à creche e estado nutricional de pré-escolares: uma revisão sistemática. Rev Paul Pediatr. 2010; 28 (4): 366-72.

42. Menezes TN, Rocha FL, Belem PLO, Pedraza DF. Obesidade abdominal: revisão crítica das técnicas de aferição e dos pontos de corte de indicadores antropométricos adotados no Brasil. Ciênc Saúde Coletiva. 2014; 19 (6): 1741-54. 
43. Figueroa Pedraza D, Queiroz D, Sales MC. Doenças infecciosas em crianças pré-escolares brasileiras assistidas em creches. Ciênc Saúde Coletiva. 2014; 19 (2): 511-28.

44. Anjos LA, Souza DR, Rossato SL. Desafios na medição quantitativa da ingestão alimentar em estudos populacionais. Rev Nutr. 2009; 22 (1): 151-61.

45. Silva TA, Vasconcelos SML. Procedimentos metodológicos empregados em questionários de frequência alimentar elaborados no Brasil: uma revisão sistemática. Rev Nutr. 2012; 25 (6): 785-97.

46. Cozzolino SMF, Colli C. Novas recomendações de nutrientes: interpretação e utilização. In: International Life Sciences Institute do Brasil. Usos e aplicações das "Dietary Reference Intakes” DRIs. São Paulo: ILSI; 2001. p. 5-16.

47. Instituto Brasileiro de Geografia e Estatística. Pesquisa de Orçamentos Familiares 2008-2009: análise do consumo alimentar pessoal no Brasil. Rio de Janeiro: IBGE; 2011.
48. Rinaldi AEM, Pereira AF, Macedo CS, Mota JF, Burini RC. Contribuições das práticas alimentares e inatividade física para o excesso de peso infantil. Rev Paul Pediatr. 2008; 26 (3): $271-7$.

49. Chaves LG, Santana TCM, Gabriel CG, Vasconcelos FA. Reflexões sobre a atuação do nutricionista no Programa Nacional de Alimentação Escolar no Brasil. Ciênc Saúde Coletiva. 2013; 18 (4): 917-26.

50. Oliveira MC, Vassimon HS. Programa Nacional de Alimentação Escolar e sua aceitação pelos alunos: uma revisão sistemática. Investigação. 2012; 12: 4-1.

51. Fidelis CMF, Osório MM. Consumo alimentar de macro e micronutrientes de crianças menores de cinco anos no Estado de Pernambuco, Brasil. Rev Bras Saúde Matern Infant. 2007; 7 (1): 63-74.

Recebido em 28 de agosto de 2014

Versão final apresentada em 29 de dezembro de 2014

Aprovado em 5 de janeiro de 2015 